\title{
A case with neurogenic pulmonary edema
}

\author{
Uzar T ${ }^{1}$, Aslan $\mathrm{M}^{1}$, Celik $\mathrm{O}^{2}$, Dirican $\mathrm{A}^{2}$, Yavuz $Z^{3}$, Ekaterina $\mathrm{P}^{4}$ and Ozkaya $\mathrm{S}^{5}$ \\ ${ }^{1}$ Bahcesehir University, Faculty of Medicine, Istanbul, Turkey \\ ${ }^{2}$ Samsun Medicalpark Hospital, Department of Neurosurgery, Samsun, Turkey \\ ${ }^{3}$ Ankara University, Faculty of Medicine, Department of Neurology, Ankara, Turkey \\ ${ }^{4}$ Bashkir State Medical University, Faculty of Medicine, Department of Pulmonary Medicine, Ufa, Russia \\ ${ }^{5}$ Bahcesehir University, Faculty of Medicine, Department of Pulmonary Medicine, Istanbul, Turkey
}

\begin{abstract}
Fibrolamellar hepatocellular carcinoma (FL-HCC) is an uncommon histological subtype of Hepatocellular carcinoma, which seldom invades the gall-bladder (GB). Here we report a case of FL-HCC invading the GB, that resembled gall bladder carcinoma invading the liver on both US and CT. A central calcification and/or central stellate scar that are clue to the diagnosis of FL-HCC were absent.
\end{abstract}

\section{Introduction}

Neurogenic pulmonary edema (NPE) is a relatively rare and underdiagnosed clinical entitiy which is an increase in pulmonary interstitial and alveolar fluid. NPE develops within a few hours after a neurologic insult including spinal cord injury, subarachnoid hemorrhage (SAH), traumatic brain injury (TBI), intracranial hemorrhage, status epilepticus, meningitis, and subdural hemorrhage, have been associated with this syndrome [1-6]. We aimed to present a patient who developed NPE following subarachnoid haemorrhage.

\section{Case Presentation}

A 33-year-old man with no history of any significant past medical illness, admitted to the emergency room sudden onset fainting and loss of consciousness. CT scan of the head revealed the massive subarachnoid blood which consistent with subarachnoid haemorrhage (Figure 1A and 1B).

Shortly after admission, respiratory failure was developed with tachypnea and tachycardia. He was intubated and hemorrhagic pulmonary fluid was aspirated from endotracheal tube. Chest roentgenography showed the nonspecific, bilateral, rather

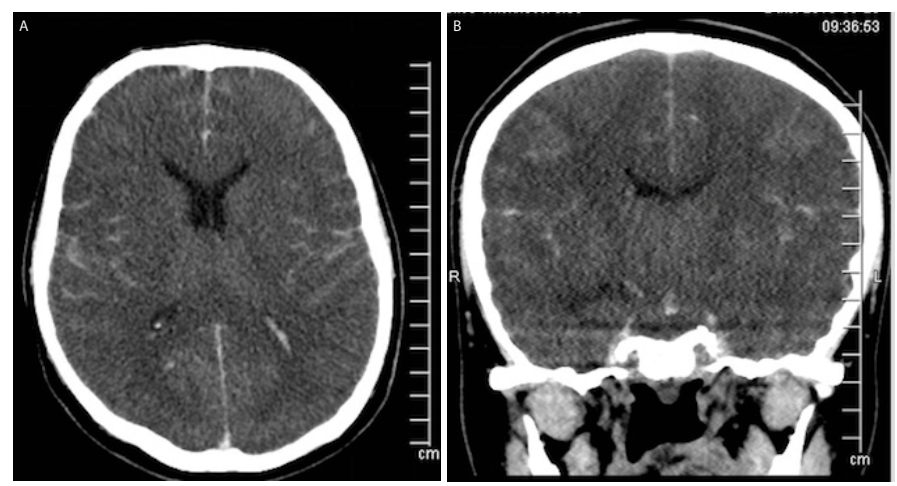

Figure 1. CT scan of the head showing the massive subarachnoid blood which consistent with subarachnoid haemorrhage homogeneous airspace consolidative appearances with an apical predominance which supported the non-cardiogenic pulmonary edema (Figure 2).

Thoracic CT scans demonstrated the bilaterally diffuse alveolar edema which consistent with neurogenic pulmonary edema (Figures 3 and 4$)$.

The pulmonary edema recovered after 24 hours of IV fluid and furosemid treatment (Figure 5).

\section{Discussion}

NPE is a potential early contributor to the pulmonary dysfunction. It develops within a few hours and caused by a myriad of CNS insults that raises Intracranial Pressure(ICP). Prevailing view is that a rapid elevation of ICP leads to neuronal compression, ischemia or damage; followed by an autonomic response. Sympathetic nervous system activation leads to release of catecholamines that results in cardiopulmonary dysfunction. The cellular mechanisms that cause capillary leakage are also not well understood. Modifications in neurovegetative pathways are probably the cause of sudden, significant increases in microvascular pressure in the lungs, particularly in the pulmonary venules. This leads to reduced venous outflow, which in turn causes pulmonary capillary and arterial hypertension [7]. Sudden onset of respiratory distress is a chief feature of NPE. Due to lack of treatment modalities, management of NPE consist of treating the underlying neurologic condition. Neurogenic pulmonary edema is described clinically as

${ }^{*}$ Correspondence to: Ozkaya S, Pulmonologist, Bahcesehir University, Faculty of Medicine, Department of Pulmonary Medicine, Istanbul, Turkey, Tel: +905324741309; E-mail: ozkayasevket@yahoo.com

Key words: neurogenic pulmonary edema, subarachnoid haemorrhage, noncardiogenic, pulmonary edema

Received: January 07, 2019; Accepted: January 18, 2019; Published: January 21 2019 


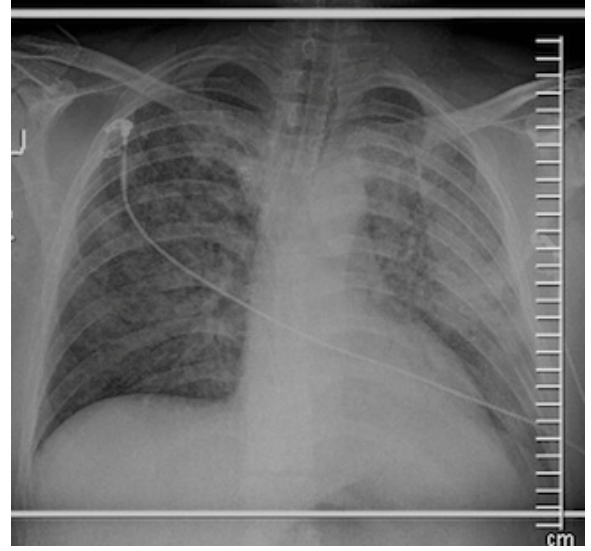

Figure 2. Chest roentgenography showing the nonspecific, bilateral, rather homogeneous airspace consolidative appearances with an apical predominance which supported the noncardiogenic pulmonary edema

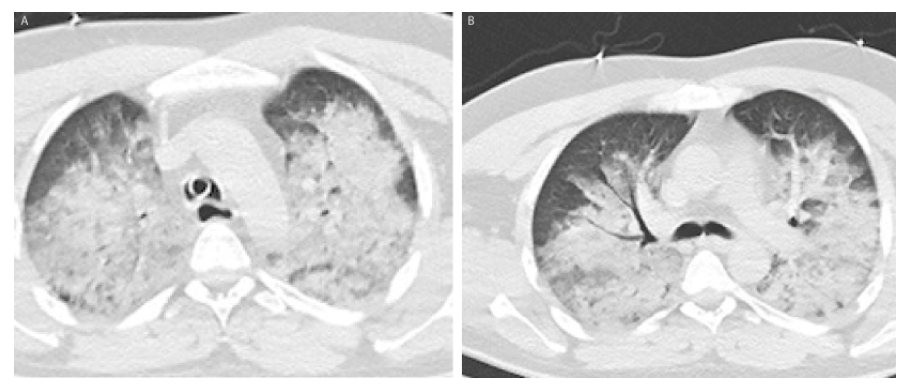

Figure 3A and 3B. The vertical section thoracic CT scans showing the bilaterally diffuse alveolar edema which consistent with neurogenic pulmonary edema(A and $\mathrm{B}$ )

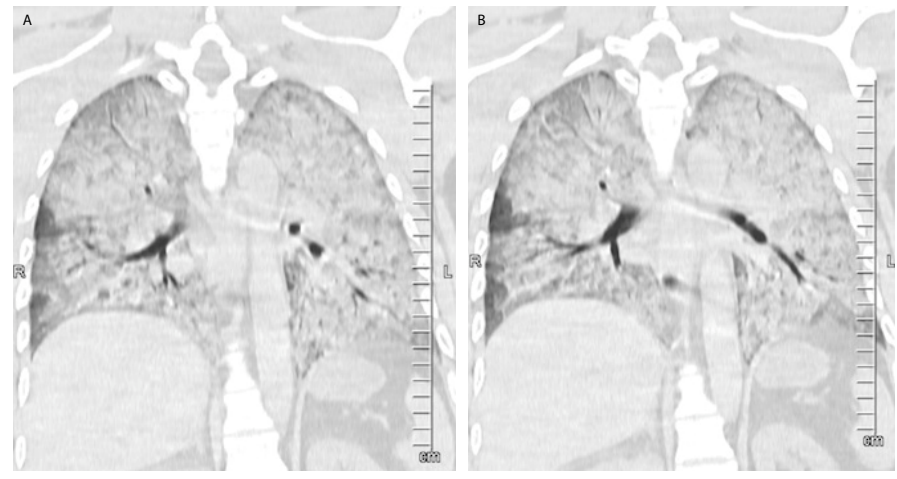

Figure 4. The coronal section thoracic $\mathrm{CT}$ scans showing the bilaterally diffuse alveolar edema (A and B)

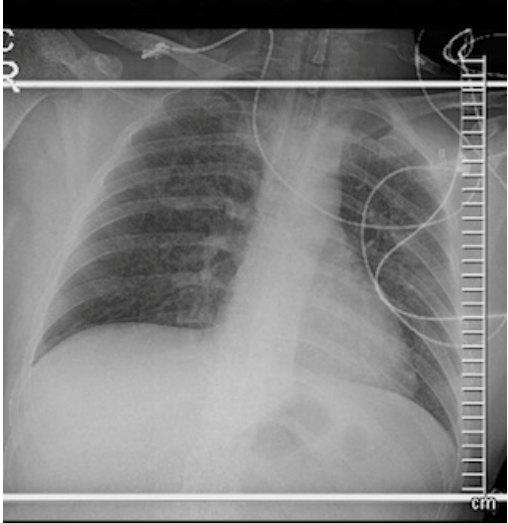

Figure 5. Chest radiograhy showing to the recorvered pulmonary edema after the treatment

sudden onset breathlessness, tachypnoea, tachycardia, hemoptysis, bilateral lung crackles on auscultation, low oxygen saturation on arterial blood gas analysis. Neurogenic pulmonary edema seen as mostly homogenous, bilateral airspace consolidations(diffuse opacities) that predominate at the apices in about half of the cases, as seen in our case. Disappearance of radiologic findings in 1-2 days supports the absence of any diffuse alveolar damage [1-7].

In conclussion; NPE is acute onset and life-threatening complication especially in patients with subarachnoid hemorrhage. If diagnosed immediately, the rapid response to treatment can be obtained.

\section{Disclosure}

The authors have no conflicts of interest to declare.

\section{References}

1. Simmons RL, Heisterkamp CA, Collins JA, Genslar S, Martin AM Jr (1969) Respiratory insufficiency in combat casualties. Arterial hypoxemia after wounding. Ann Surg 170: 45-52. [Crossref]

2. Rogers FB, Shackford SR, Trevisani GT, Davis JW, Mackersie RC, et al. (1995) Neurogenic pulmonary edema in fatal and nonfatal head injuries. J Trauma 39: 860866. [Crossref]

3. Fontes RB, Aguiar PH, Zanetti MV, Andrade F, Mandel M, et al. (2003) Acute neurogenic pulmonary edema: case reports and literature review. J Neurosurg Anesthesiol 16: 144-150. [Crossref]

4. Kaufman HH, Timberlake G, Voelker J, Pait TG (1993) Medical complications of head injury. Med Clin North Am 77: 43-60. [Crossref]

5. Colice GL (1985) Neurogenic pulmonary edema. Clin Chest Med 6: 473-489. [Crossref]

6. Davison DL, Terek M, Chawla LS (2012) Neurogenic pulmonary edema. Crit Care 16 212. [Crossref]

7. Agrawal Ai Timothy J, Pandi L (2007) Neurogenic Pulmonary Oedema. Eur J Gen Med 4: 25-32.

Copyright: (C2019 Uzar T. This is an open-access article distributed under the terms of the Creative Commons Attribution License, which permits unrestricted use, distribution, and reproduction in any medium, provided the original author and source are credited. 